\title{
Regularization scheme independence and unitarity in QCD cross sections
}

\author{
S. Catani \\ INFN, Sezione di Firenze and Dipartimento di Fisica, Università di Firenze, Largo E. Fermi 2, I-50125 Florence, Italy \\ M. H. Seymour* \\ Theory Division, CERN, CH-1211 Geneva 23, Switzerland \\ Z. Trócsányi \\ Department of Theoretical Physics, KLTE, H-4010 Debrecen, P.O. Box 5, Hungary
}

(Received 1 November 1996)

\begin{abstract}
When calculating next-to-leading order QCD cross sections, divergences in intermediate steps of the calculation must be regularized. The final result is independent of the regularization scheme used, provided that it is unitary. In this paper we explore the relationship between regularization scheme independence and unitarity. We show how the regularization scheme dependence can be isolated in simple universal components, and how unitarity can be guaranteed for any regularization prescription that can consistently be introduced in one-loop amplitudes. Finally, we show how to derive transition rules between different schemes without having to do any loop calculations. [S0556-2821(97)03611-4]

PACS number(s): 12.38.Bx, 13.87.-a
\end{abstract}

\section{INTRODUCTION}

Two major bottlenecks hamper the straightforward (but toilsome) implementation of QCD perturbation theory in physical computations. The first that one encounters in producing new QCD calculations for a certain process is the evaluation of the relevant matrix elements. The second regards the use of those matrix elements in the actual computation of physical quantities. Recent years have witnessed much progress in perturbative QCD calculations and both these major bottlenecks have been greatly reduced. On the one hand, new techniques have been developed to evaluate QCD amplitudes at one loop [1] and on the other, completely general, process-independent methods [2-7] have been set up to compute physical cross sections at next-to-leading order (NLO).

In particular, reasonably compact expressions for all fiveparton one-loop amplitudes have been obtained [8-10] and NLO calculations for physical processes involving many partons have become feasible. The three-jet cross section in hadron collisions in the simplified case of pure-gluon subprocesses is now available [11,12], as is the four-jet cross section in electron-positron annihilation in the simplified cases of leading color [13] and four-quark final states [14]; the full QCD results are expected to appear soon.

These two bottlenecks are, however, strongly related, and overcoming them independently is not sufficient for a successful implementation of perturbative QCD. The relationship is due to the regularization of unphysical divergences and, ultimately, to unitarity.

Theoretical evaluation of jet observables, i.e., infrared and collinear safe quantities, should lead to unambiguous results for physical cross sections. As a statement of principle this is

\footnotetext{
*Present address: Rutherford Appleton Laboratory, Chilton, Didcot, Oxfordshire, OX11 0QX, United Kingdom.
}

almost trivial, but its practical realization is far from trivial. This is because expressions at intermediate steps of the calculation (loop matrix elements and integrals of tree-level matrix elements) contain ultraviolet, soft, and collinear divergences. Consequently, one has to introduce some regularization procedure. Eventually, the regularized singularities cancel in physical quantities and the finite remainder should be independent of the regularization procedure. This independence is only achieved, however, if the regularization prescription is unitary. The evaluation of the matrix elements and their implementation in the calculation of physical observables must therefore be carried out without violating unitarity.

A regularization scheme (RS) that fulfills the requirement of unitarity in QCD is known, conventional dimensional regularization. However, the intermediate ingredients that are necessary for a complete evaluation of cross sections are not always available within this RS. In fact, the new techniques for calculating one-loop QCD amplitudes use a different version of dimensional regularization, dimensional reduction [15]. Moreover, even when the squared matrix elements in conventional dimensional regularization (or any other unitary RS) are available, calculations in other schemes may be simpler. Thus it is worth while investigating whether the RS dependence can be controlled in a simple way.

Within the context of dimensional regularization, the RS dependence of one-loop amplitudes has been studied in detail. In Ref. [16], from the general structure of the squared matrix elements for all $2 \rightarrow 2$ QCD subprocesses, effective transition rules were derived to relate the NLO loop corrections in conventional dimensional regularization to those in several different dimensional regularization schemes, including dimensional reduction. For practical purposes, this information on the matrix elements is sufficient for most of the QCD computations at NLO.

Using a mass regularization scheme, many subtleties related to a consistent unitary implementation of QCD ampli- 
tudes in cross-section calculations have been pointed out in Ref. [17] for the specific case of three-jet production in $e^{+} e^{-}$annihilation. The treatment of more complicated processes along the lines of Ref. [17] is far from trivial.

The general algorithm for computing jet cross sections presented in Ref. [6] provides a very simple and transparent way to study the RS independence and unitarity of QCD cross sections. In this paper we explore and illustrate some of the issues. Our main results are the following.

We show how the several RS-dependent ingredients can explicitly be isolated in simple contributions to the NLO cross sections. These contributions are universal (they do not depend on either the process or the jet quantity) and provide an explicit control on the RS independence of the calculation.

In the general context of dimensional regularization, we derive the explicit RS dependence of the one-loop QCD amplitudes in a simple way without doing any loop calculations. In particular, we confirm the transition rules obtained in Ref. [16].

More generally, we can provide an explicit and simple recipe to guarantee unitarity of cross-section calculations for any regularization prescription that is consistently defined at the level of one-loop matrix elements.

As for the last point, we should point out that the RS issue considered in this paper does not regard the ultraviolet behavior. Ultraviolet divergences have to be properly regularized and then renormalized in off-shell Green functions. This leads to the introduction of the running coupling $\alpha_{S}\left(\mu^{2}\right)$, which we always assumed to be defined in a fixed renormalization scheme, say, the modified minimal subtraction $\overline{\mathrm{MS}}$ scheme (the renormalization-scheme dependence can always be controlled by an overall perturbative shift in $\alpha_{S}$ ). The on-shell limit of the Green functions thus defines the singular (because of soft and collinear divergences) loop amplitudes we are concerned with. These singularities are unphysical in the sense that they disappear in any jet observable, i.e., in any physical quantity that is well-defined in QCD perturbation theory. Thus there is no need to consider only (soft and collinear) regularization prescriptions that are completely justified on a field theoretical basis (for instance, to any perturbative order or for both three-level and loop amplitudes). One can introduce any regularization prescription that is well-defined at the sole level of loop amplitudes: by explicit construction, we shall show how one can then enforce unitarity in the cross-section calculation at NLO.

The outline of the paper is as follows. In Sec. II we recall the general and precise definitions of the NLO QCD cross sections we aim to calculate, and discuss their relationship with the unitarity condition. In Sec. III we recall the important features of a general formalism - the dipole formalism [6] - to calculate those cross sections. We identify the terms that may contain RS dependence, and write down the explicit conditions that ensure unitarity of physical cross sections for any RS that is consistently defined at the level of one-loop matrix elements. In Sec. IV we show that, within the general framework of dimensional regularization, the explicit RS dependence of NLO QCD calculation can be derived from that of the corresponding Altarelli-Parisi splitting functions. We do that for the main schemes in current usage. Sec. V contains our conclusions.

\section{QCD CROSS SECTIONS AT NEXT-TO-LEADING ORDER}

According to the QCD factorization theorem, the hadronlevel cross section for a jet observable has the following expression:

$$
\sigma_{\text {had }}=f \times \sigma \times d,
$$

where $\sigma$ is the parton-level cross section and $f$ and $d$ are the nonperturbative parton densities and parton fragmentation functions of the incoming and observed (in the final state) hadrons, respectively. The notation in Eq. (1) is symbolic (see, for instance, Sec. VI of Ref. [6] for a detailed notation). The hadronic cross section $\sigma_{\text {had }}$ depends on the definition of the jet quantity and on the momenta of initial-state and finalstate observed particles. The parton-level cross section depends on the jet definition, on the parton momenta and on the parton flavors. The crosses in Eq. (1) stand for the convolution over the momentum fractions and for the sum over the flavors.

We recall that $\sigma_{\text {had }}$ is a physical cross section while $f$, $d$, and $\sigma$ are not separately physical quantities. They depend on the factorization scheme. Having defined this scheme, i.e., the process-independent parton distributions $f$ and $d$, the partonic cross section $\sigma$ is computable with no ambiguities in perturbation theory to any order in $\alpha_{s}$. Its perturbative expansion up to NLO is

$$
\sigma=\sigma^{\mathrm{LO}}+\sigma^{\mathrm{NLO}} .
$$

The leading order (LO) contribution $\sigma^{\mathrm{LO}}$ is obtained by integrating the fully exclusive cross section $d \sigma^{B}$ in the Born approximation over the phase space for the corresponding jet quantity. Suppose that this LO calculation involves $m$ partons in the final state. Thus, we write

$$
\sigma^{\mathrm{LO}}=\int_{m} d \sigma^{B}
$$

Note that the LO cross section in Eq. (3) is finite by definition.

Using analogous notation, the NLO cross section $\sigma^{\mathrm{NLO}}$ is a sum of three integrals:

$$
\sigma^{\mathrm{NLO}}=\int_{m+1} d \sigma^{R}+\int_{m} d \sigma^{V}+\int_{m} d \sigma^{C} .
$$

Here $d \sigma^{C}$ is a counterterm that defines the factorization scheme, $d \sigma^{R}$ (the "real" cross section) is the exclusive cross section with $m+1$ partons in the final state, and $d \sigma^{V}$ (the "virtual" cross section) is the one-loop correction to the process with $m$ final-state partons.

Strictly speaking, the expression on the right-hand side of Eq. (4) has only a formal meaning because its contributions are separately divergent. The virtual cross section $d \sigma^{V}$ is proportional to the one-loop matrix element that, although renormalized, still contains soft and collinear singularities coming from the loop integration. In order to remove these infinities, the loop integral has to be regularized and, correspondingly, $d \sigma^{V}$ is replaced by its (RS-dependent) regularized version $d \sigma_{\mathrm{RS}}^{V}$. The real cross section $d \sigma^{R}$ is finite but 
its integration over the $(m+1)$-parton phase space produces soft and collinear divergences that cancel those in $d \sigma^{V}$, thus leading to a finite NLO cross section ${ }^{1} \sigma^{\mathrm{NLO}}$. This finite remainder is unambiguously defined provided that, before its integration, the real cross section $d \sigma^{R}$ is replaced by a consistently regularized version $d \sigma_{\mathrm{RS}}^{R}$. A more correct way of writing Eq. (4) is, thus,

$$
\sigma^{\mathrm{NLO}}=\int_{m+1} d \sigma_{\mathrm{RS}}^{R}+\int_{m} d \sigma_{\mathrm{RS}}^{V}+\int_{m} d \sigma^{C},
$$

where some formal limit RS $\rightarrow 0$ is understood.

Consistency of the RS means unitarity. More precisely, unitarity of the regularized theory implies that the scattering amplitudes $T_{a b}^{\mathrm{RS}}$ that are used to compute its matrix elements have to fulfill the following unitarity condition:

$$
2 \operatorname{Im} T_{a a}^{\mathrm{RS}}=\sum_{b}\left|T_{a b}^{\mathrm{RS}}\right|^{2},
$$

up to the relevant order in perturbation theory. In particular, at NLO the discontinuity of the one-loop matrix element (which is used in $d \sigma_{\mathrm{RS}}^{V}$ ) on the left-hand side of Eq. (6) provides a constraint on the squares of the tree-level matrix elements (which are used in $d \sigma_{\mathrm{RS}}^{R}$ ) on the right-hand side.

The unitarity condition in Eq. (6), which ultimately is at the basis of the cancellation theorems [18], also shows that the main difficulty in controlling the cancellation of divergences in Eq. (4) has a kinematic origin. If we had to compute the total cross section for a given process, the real and virtual contributions could be combined at the integrand level before doing the loop integral. In the soft and collinear regions, this integrand would contain exactly the difference between the left- and right-hand sides of Eq. (6) and, hence, would be integrable even without introducing any regularization prescription. On the contrary, in Eq. (4) the integrations of the real and virtual contributions have to be performed over different phase-space regions. The shape of the two phase-space regions depends in a nontrivial way both on the number of partons and on the actual definition of the cross section. In principle, one has to do a detailed calculation for any different jet observable in any given process.

A general strategy [2-7] to overcome this difficulty consists in trying to expose the cancellation of singularities directly at the integrand level. This amounts to recasting Eq. (4) in the following form:

$$
\sigma^{\mathrm{NLO}}=\sigma^{\mathrm{NLO}\{m+1\}}+\hat{\sigma}^{\mathrm{NLO}\{m\}}+\sigma^{\mathrm{NLO}\{m\}} .
$$

In the contribution $\sigma^{\mathrm{NLO}\{m+1\}}$, the integration is carried out over the $(m+1)$-parton phase space. The contributions $\sigma^{\mathrm{NLO}\{m\}}$ and $\hat{\sigma}^{\mathrm{NLO}\{m\}}$, instead, involve the integration over the $m$-parton phase space.

\footnotetext{
${ }^{1}$ The integral of $d \sigma^{R}$ also produces additional collinear divergences that cancel those in $d \sigma^{C}$. Although these are in fact regularized by the same scheme as the others, the resulting RS dependence can be absorbed into the factorization scheme dependence of $d \sigma^{C}$, which does not concern us here.
}

The precise definition of these three terms depends on the detailed method used in going from Eq. (4) to Eq. (7). The common feature is that the three integrands are separately finite. More precisely, the integrand in $\sigma^{\mathrm{NLO}\{m+1\}}$ is explicitly RS independent in the sense that it can be defined without introducing any regularization scheme. The integrand in $\hat{\sigma}^{\mathrm{NLO}\{m\}}$ is also explicitly RS independent, although it depends on the factorization scheme. The integrand in $\sigma^{\mathrm{NLO}\{m\}}$ contains the sum of two terms: they are separately divergent if the regularization is removed. Both the divergences and the RS dependence (should) cancel in the sum. In the rest of this paper, we concentrate on the finiteness and RS dependence of the integrand in $\sigma^{\mathrm{NLO}\{m\}}$.

\section{DIPOLE FORMALISM AND THE ISSUE OF UNITARITY}

The dipole factorization formulas and the general algorithm for computing QCD cross sections presented in Refs. $[5,6]$ are particularly convenient for studying the RS dependence of the integrand in $\sigma^{\mathrm{NLO}\{m\}}$. They indeed provide completely general and explicit expressions for the crosssection contributions on the right-hand side of Eq. (7). In particular, all the kinematic complications related to the actual definition of the jet quantity are confined in overall factors.

The key point of the dipole formalism is the universal definition of a "fake" cross section $d \sigma_{\mathrm{RS}}^{A}$ that depends on the momenta of the $m+1$ real partons involved in the evaluation of the NLO cross section in Eq. (4). The fake cross section has the general form

$$
d \sigma_{\mathrm{RS}}^{A}=\sum_{\text {dipoles }} d \sigma_{\mathrm{RS}}^{B} \otimes d V_{\mathrm{dipole}}^{\mathrm{RS}} .
$$

In Eq. (8) the only dependence on the physical quantity we are interested in is contained in the factor $d \sigma_{\mathrm{RS}}^{B}$. This is exactly the (regularized version of) Born-level cross section that enters in the calculation of the $\mathrm{LO}$ cross section $\sigma^{\mathrm{LO}}$ in Eq. (3). The only other ingredients needed to construct $d \sigma_{\mathrm{RS}}^{A}$ are the dipole factors $d V_{\text {dipole. }}^{\mathrm{RS}}$. They depend on the RS but are otherwise universal: the dipole factors are completely process and observable independent and can be given once and for all [6] starting from the regularized $S$ matrix of the theory. The symbol $\otimes$ denotes properly defined correlations between colors and helicities of the partons in $d \sigma_{\mathrm{RS}}^{B}$ and in the dipole factors (see Sec. IV A).

The first main property of Eq. (8) is that, in the soft and collinear regions, $d \sigma_{\mathrm{RS}}^{A}$ has the same pointwise singular behavior as the (regularized) real cross section $d \sigma_{\mathrm{RS}}^{R}$ in Eq. (5)

$$
d \sigma_{\mathrm{RS}}^{R} \underset{\left(\begin{array}{c}
\text { soft and/or } \\
\text { collinear }
\end{array}\right)}{\longrightarrow} d \sigma_{\mathrm{RS}}^{A} .
$$

Equation (9) follows from the factorizing properties [19] of soft and collinear radiation in gauge theories and the dipole factorization theorem introduced in Ref. [5]. The dipole factors are precisely defined [6] by the emission probability of soft and collinear partons.

There are several dipole terms on the right-hand side of Eq. (8). Each of them corresponds to a different kinematic 
configuration of the $m+1$ real partons. Each configuration can be thought of as obtained by an effective two-step process: an $m$-parton configuration is first produced and then one of these partons decays into two partons. The Born-level cross section $d \sigma_{\mathrm{RS}}^{B}$ depends on the $m$-parton configuration, and the dipole factors describe the one-to-two parton decays. This two-step pseudoprocess can be defined without introducing any approximation on the $(m+1)$-parton kinematics, thus leading to the second main property of Eq. (8): exact factorization of the phase space [6].

Exact factorization means that we can carry out a factorizable mapping from the $(m+1)$-parton phase space to an $m$-parton subspace, identified by the partonic variables in $d \sigma_{\mathrm{RS}}^{B}$, times a single-parton phase space, identified by the dipole partonic variables in $d V_{\text {dipole. This mapping makes }}^{\mathrm{RS}}$ $d V_{\text {dipole }}^{\mathrm{RS}}$ fully integrable analytically. We can write:

$$
\int_{m+1} d \sigma_{\mathrm{RS}}^{A}=\sum_{\text {dipoles }} \int_{m} d \sigma_{\mathrm{RS}}^{B} \otimes \int_{1} d V_{\mathrm{dipole}}^{\mathrm{RS}}=\int_{m}\left[d \sigma_{\mathrm{RS}}^{B} \otimes \mathbf{I}^{\mathrm{RS}}\right],
$$

where the universal factor $\mathbf{I}^{\mathrm{RS}}$ is defined by

$$
\mathbf{I}^{\mathrm{RS}}=\sum_{\text {dipoles }} \int_{1} d V_{\text {dipole }}^{\mathrm{RS}}
$$

These two main properties of $d \sigma_{\mathrm{RS}}^{A}$ allow us to obtain the decomposition in Eq. (7) by a straightforward implementation of the subtraction method [20]. The fake cross section in Eq. (8) can be subtracted from $d \sigma_{\mathrm{RS}}^{R}$ and $d \sigma^{C}$, and then added back to the right-hand side of Eq. (5). This subtraction defines the integrands of $\sigma^{\mathrm{NLO}\{m+1\}}$ and $\hat{\sigma}^{\mathrm{NLO}\{m\}}$ in Eq. (7), which, due to Eq. (9), are explicitly RS independent, since the RS can be removed already in the integrand. The remaining term defines $\sigma^{\mathrm{NLO}\{m\}}$. Indeed, combining the virtual cross section $d \sigma_{\mathrm{RS}}^{V}$ with Eq. (10), we can write this contribution as

$$
\sigma^{\mathrm{NLO}\{m\}}=\int_{m}\left[d \sigma_{\mathrm{RS}}^{V}+d \sigma_{\mathrm{RS}}^{B} \otimes \mathbf{I}^{\mathrm{RS}}\right]_{\mathrm{RS}=0} .
$$

The second term in the square bracket of Eq. (12) contains all the regularized singularities that are necessary to cancel the (equal and with opposite sign) regularized singularities in the virtual correction $d \sigma_{\mathrm{RS}}^{V}$. After adding these two terms, one can thus remove the regularization (as implied by the notation $\mathrm{RS}=0$ ).

Actually, as explicitly denoted on the right-hand side of Eq. (12), the regularization can be removed before carrying out the integration over the $m$-parton phase space. The reason for this is the following. First, as recalled below Eq. (4), soft and collinear divergences in $d \sigma_{\mathrm{RS}}^{V}$ arise from the loop integral in the one-loop matrix element, independently of the definition of the virtual cross section. Secondly, in the second term on the right-hand side of Eq. (12) the dependence on the cross-section kinematics is fully contained in $d \sigma_{\mathrm{RS}}^{B}$ which, as pointed out below Eq. (3), is integrable by definition. Following this observation, Eq. (12) can be rewritten so as to eliminate all the kinematic dependence from our discussion.
The virtual cross section $d \sigma_{\mathrm{RS}}^{V}$ can be written as

$$
d \sigma_{\mathrm{RS}}^{V}=\sum_{\{m\}} d \Phi^{(m)}(\{p\})\left|\mathcal{M}^{\mathrm{RS}}(\{p\})\right|_{1 \text { loop }}^{2},
$$

where $\Sigma_{\{m\}}$ stands for the sum over all the configurations with $m$ partons, $\left|\mathcal{M}^{\mathrm{RS}}(\{p\})\right|_{1 \text { loop }}^{2}$ is the one-loop matrix element squared, and $\{p\}$ denotes its dependence on the parton momenta (these include the $m$ final-state partons as well as possible partons in the initial state). The factor $d \Phi^{(m)}(\{p\})$ in Eq. (13) contains all the other contributions to the differential cross section: spin and color average factors, the $m$-parton phase space, and, in particular, the explicit definition of the jet observable in terms of the parton momenta $\{p\}$.

The Born-level differential cross section $d \sigma_{\mathrm{RS}}^{B}$, being itself defined on the $m$-parton phase space, has exactly the same form as Eq. (13), apart from replacing the one-loop matrix element $\left|\mathcal{M}^{\mathrm{RS}}\right|_{1 \text { loop }}^{2}$ with the corresponding tree-level matrix element $\left|\mathcal{M}^{\mathrm{RSS}}\right|^{2}$. Thus, inserting Eq. (13) and the analogous expression for $d \sigma_{\mathrm{RS}}^{B}$ into Eq. (12), we obtain

$$
\sigma^{\mathrm{NLO}\{m\}}=\int \sum_{m\{m\}} d \Phi^{(m)}(\{p\}) \mathcal{F}(\{p\}),
$$

where (omitting the notation $[\cdots]_{\mathrm{RS}=0}$, from now on) we have introduced the following quantity:

$$
\mathcal{F}(\{p\})=\left|\mathcal{M}^{\mathrm{RS}}(\{p\})\right|_{1 \text { loop }}^{2}+\left|\mathcal{M}^{\mathrm{RS}}(\{p\})\right|^{2} \otimes \mathbf{I}^{\mathrm{RS}}(\{p\}) .
$$

The core of Eq. (14), namely the function $\mathcal{F}(\{p\})$, embodies all the relevant RS information. Although obtained by adding the two separately divergent and RS-dependent terms on the right-hand side of Eq. (15), this function is finite and RS independent in any unitary RS.

Equation (15) is our master equation for the study of unitarity and RS independence of physical cross sections. It can be considered as the analogue of the unitarity condition (6). From a field-theory viewpoint, Eq. (15) is not as basic as Eq. (6). Nonetheless, it provides us with an explicit implementation of Eq. (6) directly at the level of cross-section calculations and thus, it can be quite useful in practical terms. In order to discuss this point, let us consider the main features of Eq. (15).

The master function $\mathcal{F}(\{p\})$ is universal. It controls the soft and collinear singularities of physical cross sections but depends on process- and observable-independent contributions, the one-loop and tree-level matrix elements and the integral of the dipole factors. This makes explicit the statement in the first item on the list in Sec. I.

Within the context of the dipole formalism and the general algorithm for computing QCD cross sections of Ref. [6], Eq. (15) allows a straightforward implementation of different RS's in actual calculations of physical quantities.

In general, one can use Eq. (15) as a simple recipe to enforce and guarantee unitarity and RS independence in the evaluation of physical cross sections. As recalled in Sec. I, progress in the computation of loop amplitudes cannot be disjoint from similar progress in the use of these amplitudes for cross-section calculations. In principle, one should regu- 
larize unphysical soft and collinear divergences by introducing a RS that is manifestly unitary, evaluate accordingly the real and virtual cross-section contributions in Eq. (4), and perform all the steps that are necessary to end up with finite physical cross sections. Equation (15) can be used as a convenient short cut for this procedure. In order to actually evaluate $\left|\mathcal{M}^{\mathrm{RS}}(\{p\})\right|_{1 \text { loop }}^{2}$, it is sufficient to introduce a regularization prescription that, at the level of one-loop amplitudes, is defined in a consistent manner (it should not spoil general properties such as gauge invariance and renormalizability). Then, one can compute the second term on the righthand side of Eq. (15) accordingly: (1) the partons in the tree-level matrix element $\mathcal{M}^{\mathrm{RS}}(\{p\})$ have to be treated like the external partons in loop amplitudes; (2) the parent parton and its (soft and collinear) decay partons in the dipole factors $d V_{\text {dipole }}^{\mathrm{RS}}$ have to be treated like the partons inside loop integrals; (3) the dipole phase space involved in the integral (11) of the dipole factors has to be treated like the phase space in the loops. These rules are sufficient to calculate the master function $\mathcal{F}(\{p\})$ that contains all the relevant dynamical information on soft and collinear divergences. Having $\mathcal{F}(\{p\})$ to hand, one can easily perform RS transformations independently of the use of the dipole formalism in actual cross-section computations.

The reason why the unitarization recipe just outlined works is simple. The integral $\mathbf{I}^{\mathrm{RS}}(\{p\})$ of the dipole factors is proportional (but with opposite sign) to the discontinuity of the one-loop amplitude in the soft and collinear regions. Therefore, any unitarity defect in the regularization of the loop integral is automatically corrected by a corresponding contribution in the dipole factors according to Eq. (6). Note that, in this respect, the concise notation in Eq. (15) may appear confusing. In fact, the tree-level amplitudes $\mathcal{M}^{\mathrm{RS}}(\{p\})$ are matrix elements of the regularized theory but do not necessarily provide a complete set of them. The matrix elements $\mathcal{M}^{\mathrm{RS}}(\{p\})$ are evaluated over the customary partonic states, while the saturation of the unitarity condition (6) requires the sum over all possible states that contribute to the discontinuity of the loop amplitude. The regularization prescription of soft and collinear singularities in the loop can introduce unphysical states: ${ }^{2}$ in Eq. (15) the contribution of these is cancelled by analogous terms in the integral of the dipole factors.

Note also that our unitarization recipe does not require the actual calculation of loops. It is sufficient to integrate the dipole factors, which are tree-level objects in every respect. This is a nontrivial computational simplification.

The use of infrared regularization prescriptions that are not manifestly unitary may appear an oddity. However, there are in fact such schemes in current practice. In the case of ultraviolet divergences, for instance, an unequal treatment of particles inside the loop and external particles can easily be

\footnotetext{
${ }^{2}$ The role of these unphysical states in fulfilling the unitarity condition is somewhat analogous to that of the Faddeev-Popov (ultraviolet) ghosts. In some specific cases they act as a negative number of scalar fields, so we can call them "infrared ghosts," although in general this analogy is only heuristic. Because of the way in which we treat them, they are more like Feynman's "dopey particle", [21] than the formal ghosts of Faddeev and Popov.
}

reconciled with unitarity. A similar unequal treatment in the case of soft and collinear divergences is not so harmless. In the calculation of physical cross sections, the infrared singularities of loop amplitudes are cancelled by corresponding singularities arising from the integration of tree-level matrix elements: in the latter, it is not so trivial to make a distinction between "internal" and "external" particles. This point can be clarified by the explicit examples considered in Sec. IV in the context of dimensional-regularization prescriptions.

\section{DIMENSIONAL REGULARIZATION}

\section{A. Conventional dimensional regularization}

The RS known as conventional dimensional regularization simultaneously regularizes ultraviolet $[22,23]$ and soft and collinear divergences [24]. It amounts to analytically continuing parton momenta to $d=4-2 \epsilon$ space-time dimensions and to considering $d-2$ helicity states for gluons and 2 helicity states for massless quarks. No distinction is made between real and virtual partons. This RS is manifestly Lorentz and gauge invariant and consistent with unitarity.

In Ref. [6], the calculations were carried out using conventional dimensional regularization. Here we summarize the explicit results. For this purpose, we recall some notation.

It is useful to introduce a basis $\left\{\left|c_{1}, \ldots, c_{n}\right\rangle \otimes\left|s_{1}, \ldots, s_{n}\right\rangle\right\}$ in color+helicity space in such a way that the tree-level matrix element with $n$ partons can be written as

$$
\begin{aligned}
& \mathcal{M}^{c_{1}, \ldots, c_{n} ; s_{1}, \ldots, s_{n}}\left(p_{1}, \ldots, p_{n}\right) \\
& \equiv\left(\left\langle c_{1}, \ldots, c_{n}\left|\otimes\left\langle s_{1}, \ldots, s_{n}\right|\right) \mid 1, \ldots, n\right\rangle,\right.
\end{aligned}
$$

where $\left\{c_{1}, \ldots, c_{n}\right\},\left\{s_{1}, \ldots, s_{n}\right\}$, and $\left\{p_{1}, \ldots, p_{n}\right\}$ are, respectively, color indices, spin indices, and momenta of the partons. Thus $|1, \ldots, n\rangle$ is a vector in color + helicity space. ${ }^{3}$

According to this notation, the (RS-dependent) tree-level matrix element squared summed over colors and helicities is

$$
\left|\mathcal{M}^{\mathrm{RS}}(\{p\})\right|^{2}={ }_{\mathrm{RS}}\langle 1, \ldots, n \mid 1, \ldots, n\rangle_{\mathrm{RS}},
$$

while the color and spin correlations denoted by $\otimes$ in Eq. (15) (and everywhere throughout the paper) are given as

$$
\left|\mathcal{M}^{\mathrm{RS}}(\{p\})\right|^{2} \otimes \mathbf{I}^{\mathrm{RS}}(\{p\})={ }_{\mathrm{RS}}\left\langle 1, \ldots, n\left|\mathbf{I}^{\mathrm{RS}}(\{p\})\right| 1, \ldots, n\right\rangle_{\mathrm{RS}},
$$

where the integral $\mathbf{I}^{\mathrm{RS}}$ of the dipole factors is a matrix in color+helicity space and acts as an insertion operator on the right-hand side of Eq. (18).

In conventional dimensional regularization, although the dipole factors are helicity dependent, spin correlations vanish after integration over the dipole phase space. Thus, the insertion operator ${ }^{4} \mathbf{I}(\{p\}, \epsilon)$ is diagonal in the helicity space and

\footnotetext{
${ }^{3}$ In the case of initial-state partons, the definition of the state vector $|1, \ldots, n\rangle$ in Eq. (16) differs by a normalization factor (proportional to the number of colors) with respect to the definition used in Ref. [6] (cf. Eq. (3.11) in [6]).

${ }^{4}$ Since we consider conventional dimensional regularization as the default case, we drop the label RS in this scheme.
} 
depends only on the color charges $\mathbf{T}_{I}$ (see Sec. 3.2 of [6] for their detailed definition) and momenta $p_{I}$ of the partons in the tree-level matrix element $\mathcal{M}(\{p\})(I=1, \ldots, n)$. Its explicit expression is [6]

$$
\begin{aligned}
\mathbf{I}(\{p\}, \epsilon)= & -\frac{\alpha_{S}}{2 \pi} \frac{1}{\Gamma(1-\epsilon)} \sum_{I} \frac{1}{\mathbf{T}_{I}^{2}} \mathcal{V}_{I}(\epsilon) \\
& \times \sum_{J \neq I} \mathbf{T}_{I} \cdot \mathbf{T}_{J}\left(\frac{4 \pi \mu^{2}}{2 p_{I} \cdot p_{J}}\right)^{\epsilon},
\end{aligned}
$$

where $\mu$ is the dimensional-regularization scale and the singular (for $\epsilon \rightarrow 0$ ) function $\mathcal{V}_{I}(\epsilon)$ depends only on the parton flavor and has the $\epsilon$ expansion

$$
\mathcal{V}_{I}(\epsilon)=\mathbf{T}_{I}^{2}\left(\frac{1}{\epsilon^{2}}-\frac{\pi^{2}}{3}\right)+\gamma_{I} \frac{1}{\epsilon}+\gamma_{I}+K_{I}+O(\epsilon) .
$$

For present purposes there is no need to recall the detailed calculations leading to Eqs. (19) and (20). It is sufficient to note that the constants $\gamma_{I}$ and the $K_{I}$ in Eq. (20) are related to the $d$-dimensional integral of the Altarelli-Parisi splitting functions consistently evaluated in conventional dimensional regularization (cf. Sec. IV C). As a matter of fact, we have

$$
\begin{aligned}
- & \frac{1}{2} \sum_{b} \int_{0}^{1} d z[z(1-z)]^{-\epsilon}\left\langle\hat{P}_{a b}(z ; \epsilon)\right\rangle \\
& =2 \mathbf{T}_{a}^{2} \frac{1}{\epsilon}+\gamma_{a}+\left(K_{a}-\frac{\pi^{2}}{6} \mathbf{T}_{a}^{2}\right) \boldsymbol{\epsilon}+O\left(\epsilon^{2}\right),
\end{aligned}
$$

where $\left\langle\hat{P}_{a b}(z ; \epsilon)\right\rangle$ denotes the azimuthally averaged splitting function.

For the sake of completeness, the actual values of the constants entering into Eq. (20) are

$$
\begin{gathered}
\mathbf{T}_{q}^{2}=\mathbf{T}_{q}^{2}=C_{F}, \quad \mathbf{T}_{g}^{2}=C_{A}, \\
\gamma_{q}=\gamma_{\bar{q}}=\frac{3}{2} C_{F}, \quad \gamma_{g}=\frac{11}{6} C_{A}-\frac{2}{3} T_{R} N_{f}, \\
K_{q}=K_{\bar{q}}=\left(\frac{7}{2}-\frac{\pi^{2}}{6}\right) C_{F}, \quad K_{g}=\left(\frac{67}{18}-\frac{\pi^{2}}{6}\right) C_{A}-\frac{10}{9} T_{R} N_{f},
\end{gathered}
$$

where $C_{F}=\left(N_{c}^{2}-1\right) / 2 N_{c}, C_{A}=N_{c}, T_{R}=1 / 2, N_{c}$ is the number of colors and $N_{f}$ is the number of massless flavors.

\section{B. Regularization prescriptions within dimensional regularization}

Dimensional regularization was invented to regularize ultraviolet divergences in loop integrals of gauge theories in a gauge-invariant manner. In this respect the essential ingredient is the continuation of loop momenta into $d \neq 4$ dimensions. Having done this, one is left with some freedom regarding the dimensionality of the momenta of the external particles as well as the number of polarizations of both external and internal particles.

The original choice of 't Hooft and Veltman [22] was to continue the particle momenta and the helicities of vector particles inside loops into $d \neq 4$ dimensions, while keeping the momenta and helicities of external particles, as well as fermion helicities inside loops, in four dimensions. Another version of dimensional regularization, namely dimensional reduction, was introduced in Ref. [15] in order to explicitly preserve supersymmetric Ward identities in gauge theories. This dimensional regularization prescription consists of continuing the virtual momenta of loop integrals into $d$ dimensions while keeping all (internal and external) polarizations in four dimensions. Although the operational definition of dimensional reduction is not complete in some higher-loop computations [25], it has been explicitly checked [26] that gauge and supersymmetry invariance is maintained up to two-loop order. In practical one-loop calculations this prescription works like conventional dimensional regularization apart from a subtle (and important) point: one has to distinguish between four-dimensional metric tensors coming from the Lorentz algebra of the spin indices and $d$-dimensional metric tensors arising from momentum integrals with more than one-loop momentum in the numerator. As for the momenta of the external partons one can keep them in $d$ dimensions (as originally proposed and used, for instance, in Ref. [27]) or in four dimensions [16]. The latter option, in particular, is systematically used in the string-theory inspired techniques [28] for computing QCD one-loop amplitudes $[1,8-10]$ : it leads to extreme simplifications of unnecessarily cumbersome expressions arising, for instance, in conventional dimensional regularization.

All these various dimensional regularization prescriptions work as ultraviolet regulators. As for gauge invariance, to our understanding the key point is that, in the procedure of analytic continuation, there are only two possibilities for choosing the number of external polarizations. One can set them either to their four-dimensional value or to the number of polarizations inside the loop. This prevents the propagation of spurious (additional) degrees of freedom from the loop to external states. When we regularize ultraviolet singularities, these different prescriptions simply lead to different renormalization factors in off-shell Green functions and, possibly, to a perturbative shift in the definition of the renormalized running coupling.

As soon as the same prescriptions are used to regularize also soft and collinear divergences, unitarity has to be carefully considered in the evaluation of the on-shell matrix elements, as first pointed out in Ref. [16].

In summary, we are going to discuss dimensional regularization prescriptions that at the level of one-loop amplitudes are defined as follows. The parton momenta in the loop are $d$ dimensional, while the external momenta are either $d$ dimensional (conventional dimensional regularization and dimensional reduction as in [27]) or four dimensional ('t Hooft and Veltman and dimensional reduction as in $[1,16])$. Correspondingly, the number $n_{s}(g)$ of gluon polarizations in external states is either $d-2$ or 2 . The number $h_{g}$ of gluon helicity states in the loop is analytically continued to $d$ dimensions, as in conventional dimensional regularization and in the 't Hooft-Veltman prescription,

$$
h_{g}^{\mathrm{CDR}}=h_{g}^{\mathrm{HV}}=d-2=2-2 \epsilon,
$$

or kept fixed in four dimensions, as in dimensional reduction, 
TABLE I. Definitions of various regularization prescriptions of one-loop amplitudes referred to in the text.

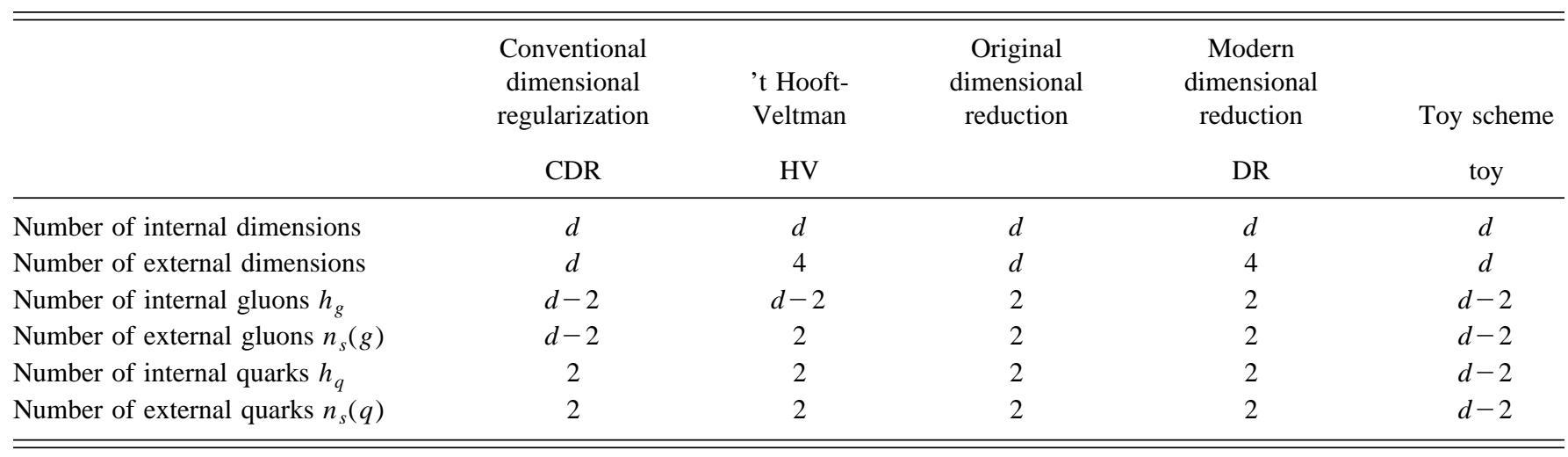

$$
h_{g}^{\mathrm{DR}}=2
$$

The number $h_{q}$ of massless-quark polarization states in the loop is 2 (as in all the regularization prescriptions discussed above) or arbitrarily continued to $d$ dimensions by $2 h_{q}=$ $\operatorname{Tr} \mathbf{1}=4+O(\epsilon), \operatorname{Tr} \mathbf{1}$ being the dimensionality of the spinor space (the definition $\operatorname{Tr} 1=4-4 \epsilon$ was used in Ref. [29]). This freedom in defining $h_{q}$ does not simplify any practical calculations and is correctly referred to as harmless in any textbook that introduces dimensional continuation as an ultraviolet regularization. In order to emphasize in the simplest way the differences between ultraviolet and infrared regulators, however, we also consider a toy scheme that is identical to conventional dimensional regularization, but with ${ }^{5} h_{q}=2$ $-2 \epsilon$. We will see that this leads to effects that, although trivial to keep track of, are not harmless. We summarize these definitions in Table I.

The implementation of these prescriptions in the master formula (15) is straightforward. Following the unitarization recipe in Sec. III, the tree-level matrix element $\mathcal{M}^{\mathrm{RS}}(\{p\})$ has to be evaluated considering the partons $\{p\}$ such as the external ones in the loop amplitude. The dipole phase space is always $d$ dimensional. The dipole factors are obtained from the emission probabilities of soft and collinear partons. However, soft emission, being gauge invariant and independent of the spin of the radiating parton, is insensitive to the treatment of the spin polarizations. Eventually, in order to relate different dimensional regularization prescriptions, we simply have to compute the corresponding Altarelli-Parisi splitting functions for collinear emission.

\section{Altarelli-Parisi splitting functions in various dimensional regularization schemes}

Let us consider the (timelike) splitting of a massless parton with flavor $a$ into two massless partons with flavors $b$ and $c$ and momenta $p_{b}$ and $p_{c}$. The collinear limit $k_{\perp} \rightarrow 0$ is precisely defined by introducing the following parametrization of the parton momenta:

\footnotetext{
${ }^{5}$ It might seem that this is the most natural scheme, since it corresponds to a theory in which every quantity is analytically continued to the same number of dimensions $d$.
}

$$
\begin{gathered}
p_{b}^{\mu}=z p^{\mu}+k_{\perp}^{\mu}-\frac{k_{\perp}^{2}}{z} \frac{n^{\mu}}{2 p \cdot n}, \\
p_{c}^{\mu}=(1-z) p^{\mu}-k_{\perp}^{\mu}-\frac{k_{\perp}^{2}}{1-z} \frac{n^{\mu}}{2 p \cdot n},
\end{gathered}
$$

where the lightlike $\left(p^{2}=0\right)$ vector $p^{\mu}$ denotes the collinear direction and $n^{\mu}$ is an auxiliary lightlike vector that is necessary to specify the transverse component $k_{\perp}\left(k_{\perp}^{2}<0, k_{\perp} p\right.$ $=k_{\perp} n=0$ ) or, equivalently, how the collinear direction is approached.

The probability of the splitting process $a \rightarrow b+c$ (summed over colors and spins of $b$ and $c$ ) in the collinear limit is proportional to the Altarelli-Parisi splitting function $\hat{P}_{a b}^{\mathrm{RS}}\left(z, k_{\perp}\right)$. Since the collinear partons $b$ and $c$ have to be treated like the partons inside loop amplitudes, this splitting function is RS dependent. Using dimensional regularization the momenta in Eq. (25) are always $d$ dimensional, but the number of polarization states of the partons $b$ and $c$ still depends on the detailed regularization prescription. Moreover, the function $\hat{P}_{a b}^{\mathrm{RS}}\left(z, k_{\perp}\right)$ depends not only on the longitudinal-momentum fraction $z$ involved in the splitting process (25) but also on the transverse momentum $k_{\perp}$ and on the helicity of the parent parton $a$ (this parton is treated like the external partons in loop amplitudes). More precisely, $\hat{P}_{a b}^{\mathrm{RS}}$ is a matrix acting on the spin indices of the parton $a$. The calculation of the polarized splitting functions is straightforward [30]. We find

$$
\begin{aligned}
& \left\langle s\left|\hat{P}_{q q}^{\mathrm{RS}}\left(z, k_{\perp}\right)\right| s^{\prime}\right\rangle=\left\langle s\left|\hat{P}_{\bar{q}}^{\mathrm{RS}}\left(z, k_{\perp}\right)\right| s^{\prime}\right\rangle \\
& =\delta_{s s^{\prime}} C_{F}\left[\frac{2 z}{1-z}+\frac{1}{2} h_{g}^{\mathrm{RS}}(1-z)\right], \\
& \left\langle s\left|\hat{P}_{q g}^{\mathrm{RS}}\left(z, k_{\perp}\right)\right| s^{\prime}\right\rangle=\left\langle s\left|\hat{P}_{\bar{q} g}^{\mathrm{RS}}\left(z, k_{\perp}\right)\right| s^{\prime}\right\rangle \\
& =\delta_{s s^{\prime}} C_{F}\left[\frac{2(1-z)}{z}+\frac{1}{2} h_{g}^{\mathrm{RS}} z\right], \\
& \left\langle\mu\left|\hat{P}_{g q}^{\mathrm{RS}}\left(z, k_{\perp}\right)\right| \nu\right\rangle=\left\langle\mu\left|\hat{P}_{g}^{\mathrm{RS}}\left(z, k_{\perp}\right)\right| \nu\right\rangle \\
& =T_{R} \frac{h_{q}}{2}\left[-g^{\mu \nu}+4 z(1-z) \frac{k_{\perp}^{\mu} k_{\perp}^{\nu}}{k_{\perp}^{2}}\right],
\end{aligned}
$$




$$
\begin{aligned}
\left\langle\mu\left|\hat{P}_{g g}^{\mathrm{RS}}\left(z, k_{\perp}\right)\right| \nu\right\rangle= & 2 C_{A}\left[-g^{\mu \nu}\left(\frac{z}{1-z}+\frac{1-z}{z}\right)\right. \\
& \left.-h_{g}^{\mathrm{RS}} z(1-z) \frac{k_{\perp}^{\mu} k_{\perp}^{\nu}}{k_{\perp}^{2}}\right],
\end{aligned}
$$

where the spin indices of the parent parton $a$ have been denoted by $s, s^{\prime}$ if $a$ is a fermion and by the Lorentz indices $\mu, \nu$ if $a$ is a gluon.

The scheme dependence related to the definition of $h_{q}$ only affects the Altarelli-Parisi function in Eq. (28) for the splitting process $g \rightarrow q+\bar{q}$. For all the other prescriptions discussed in Sec. IV B the scheme dependence is entirely parametrized by the number $h_{g}^{\mathrm{RS}}$ of gluon helicity states in the loop. This dependence consistently vanishes for $\epsilon \rightarrow 0$, i.e., if the regularization is removed. In particular, because of Eq. (23), we can immediately conclude that for the 't HooftVeltman scheme the insertion operator $\mathrm{I}^{\mathrm{RS}}(\{p\}, \epsilon)$ entering into the master formula (15) exactly coincides with that in Eq. (19) for the case of conventional dimensional regularization.

Owing to the helicity conservation in the quark-gluon vector coupling, the quark splitting functions in Eqs. (26) and (27) are diagonal in the spin indices. The gluon splitting functions in Eqs. (28) and (29) turn out to be diagonal after integration over the dipole phase space. As a matter of fact, this integration involves the azimuthal average of the $d-2$ transverse components, which gives ${ }^{6}$

$$
-\left\langle\frac{k_{\perp}^{\mu} k_{\perp}^{\nu}}{k_{\perp}^{2}}\right\rangle_{\varphi}=\frac{1}{d-2}\left(-g^{\mu \nu}+\frac{p^{\mu} n^{\nu}+n^{\mu} p^{\nu}}{p n}\right) .
$$

Inserting this expression into the tree-level matrix element as in Eq. (18), the longitudinal terms proportional to $p^{\mu}$ and $p^{\nu}$ give vanishing contributions because of the Ward identity (gauge invariance) $p^{\mu} \mathcal{M}_{\mu}^{\mathrm{RS}}=0$ and only the spin-diagonal term $-g^{\mu \nu}$ survives.

In conclusion, in any dimensional-regularization scheme the insertion operator $\mathbf{I}^{\mathrm{RS}}(\{p\}, \epsilon)$ is diagonal in the helicity space and has the same expression as the insertion operator for conventional dimensional regularization in Eq. (19):

$$
\begin{aligned}
\mathbf{I}^{\mathrm{RS}}(\{p\}, \boldsymbol{\epsilon})= & -\frac{\alpha_{S}}{2 \pi} \frac{1}{\Gamma(1-\boldsymbol{\epsilon})} \sum_{I} \frac{1}{\mathbf{T}_{I}^{2}} \mathcal{V}_{I}^{\mathrm{RS}}(\boldsymbol{\epsilon}) \\
& \times \sum_{J \neq I} \mathbf{T}_{I} \cdot \mathbf{T}_{J}\left(\frac{4 \pi \mu^{2}}{2 p_{I} \cdot p_{J}}\right)^{\boldsymbol{\epsilon}} .
\end{aligned}
$$

The RS dependence is embodied in the $\epsilon$ expansion of the flavor functions $\mathcal{V}_{I}^{\mathrm{RS}}(\epsilon)$, whose coefficients are obtained by the $d$-dimensional integration of the azimuthally averaged splitting functions $\left\langle\hat{P}_{a b}^{\mathrm{RS}}(z ; \epsilon)\right\rangle$ :

\footnotetext{
${ }^{6}$ In the case of conventional dimensional regularization the azimuthal average coincides with the average over the polarizations of the parent gluon. In other dimensional regularization prescriptions, however, the two averages are different, due to the mismatch between the number $d-2$ of transverse components and the number of helicity states of the external gluons.
}

$$
\begin{gathered}
\left\langle\hat{P}_{q q}^{\mathrm{RS}}(z ; \epsilon)\right\rangle=\left\langle\hat{P}_{\bar{q}}^{\mathrm{RS}}(z ; \epsilon)\right\rangle=C_{F}\left[\frac{2 z}{1-z}+\frac{1}{2} h_{g}^{\mathrm{RS}}(1-z)\right] \\
\left\langle\hat{P}_{q g}^{\mathrm{RS}}(z ; \epsilon)\right\rangle=\left\langle\hat{P}_{\bar{q}}^{\mathrm{RS}}(z ; \epsilon)\right\rangle=C_{F}\left[\frac{2(1-z)}{z}+\frac{1}{2} h_{g}^{\mathrm{RS}} z\right] \\
\left\langle\hat{P}_{g q}^{\mathrm{RS}}(z ; \epsilon)\right\rangle=\left\langle\hat{P}_{g \bar{q}}^{\mathrm{RS}}(z ; \epsilon)\right\rangle=T_{R} \frac{h_{q}}{2}\left[1-\frac{4}{d-2} z(1-z)\right] \\
\left\langle\hat{P}_{g g}^{\mathrm{RS}}(z ; \epsilon)\right\rangle=2 C_{A}\left[\frac{z}{1-z}+\frac{1-z}{z}+\frac{h_{g}^{\mathrm{RS}}}{d-2} z(1-z)\right]
\end{gathered}
$$

Equations (32)-(35) differ by terms of order $\epsilon$ with respect to their well-known versions [20] in conventional dimensional regularization $\left(h_{g}=d-2, h_{q}=2\right)$. According to the notation in Sec. IV A we drop the label RS in conventional dimensional regularization and parametrize the deviation from this result by the residual scheme-dependent function $\mathcal{P}_{a b}^{\mathrm{RS}}(z)$ as

$$
\left\langle\hat{P}_{a b}^{\mathrm{RS}}(z ; \epsilon)\right\rangle=\left\langle\hat{P}_{a b}(z ; \epsilon)\right\rangle+2 \epsilon \mathcal{P}_{a b}^{\mathrm{RS}}(z)+O\left(\epsilon^{2}\right) .
$$

Obviously, all the functions $\mathcal{P}_{a b}^{\mathrm{CDR}}(z)$ as well as $\mathcal{P}_{a b}^{\mathrm{HV}}(z)$ vanish.

In the toy scheme, which differs from conventional dimensional regularization simply because of the dimensionality of the $\gamma$ matrices in quark loops, the only nonvanishing $\mathcal{P}$ functions are

$$
\mathcal{P}_{g q}^{\text {toy }}(z)=\mathcal{P}_{g q}^{\text {toy }}(z)=-\frac{1}{2} T_{R}\left[z^{2}+(1-z)^{2}\right] .
$$

For dimensional reduction we have

$$
\begin{gathered}
\mathcal{P}_{q q}^{\mathrm{DR}}(z)=\mathcal{P}_{\bar{q}}^{\mathrm{DR}}(z)=C_{F} \frac{1-z}{2}, \quad \mathcal{P}_{q g}^{\mathrm{DR}}(z)=\mathcal{P}_{\bar{q}}^{\mathrm{DR}}(z)=C_{F} \frac{z}{2} \\
\mathcal{P}_{g g}^{\mathrm{DR}}(z)=C_{A} z(1-z), \quad \mathcal{P}_{g q}^{\mathrm{DR}}(z)=\mathcal{P}_{g}^{\mathrm{DR}}(z)=0
\end{gathered}
$$

In this case, some other comments are in order. Since $h_{g}^{\mathrm{DR}}$ $=2$, the quark splitting functions $\left\langle\hat{P}_{q q}^{\mathrm{DR}}(z ; \epsilon)\right\rangle$ and $\left\langle\hat{P}_{q g}^{\mathrm{DR}}(z ; \epsilon)\right\rangle$ in Eqs. (32) and (33) are actually independent of $\epsilon$, while the gluon splitting functions $\left\langle\hat{P}_{\dot{g} q}^{\mathrm{DR}}(z ; \epsilon)\right\rangle$ and $\left\langle\hat{P}_{g g}^{\mathrm{DR}}(z ; \epsilon)\right\rangle$ are not. In spite of having used only fourdimensional polarizations, the $\epsilon$ dependence enters into the gluon splitting functions through azimuthal correlations, whose average has to be carried out in $d-2$ transverse dimensions.

Note also that the difference between the splitting functions in conventional dimensional regularization and those in dimensional reduction can easily be understood on fieldtheoretical basis. As discussed in detail in Ref. [26], the relationship with conventional dimensional regularization can be investigated by splitting the four-dimensional gauge field of the dimensionally-reduced Lagrangian $\mathcal{L}^{\mathrm{DR}}$ into a 
$d$-dimensional vector $A_{\mu}$ plus $4-d=2 \epsilon$ additional components. This leads to the decomposition $\mathcal{L}^{\mathrm{DR}}=\mathcal{L}^{\mathrm{CDR}}+\mathcal{L}^{(\epsilon)}$, where $\mathcal{L}^{\mathrm{CDR}}$ is the term that involves only the $d$-dimensional vector field and coincides with the customary Lagrangian in conventional dimensional regularization. The additional $2 \epsilon$ components enter into $\mathcal{L}^{(\epsilon)}$ and behave as scalar gluons interacting with the fermion and vector fields. The ultraviolet role of these $2 \epsilon$ scalars was pointed out in Ref. [26]. For instance, the exact relation between the customary $\overline{\mathrm{MS}}$ coupling and the minimally subtracted renormalized coupling in dimensional reduction can be derived by a simple calculation as the effect of the additional $2 \epsilon$ scalars. Equation (36) displays the infrared role of these $2 \epsilon$ " "ghost states.' Indeed, the functions $\mathcal{P}_{q q}^{\mathrm{DR}}(z), \mathcal{P}_{q g}^{\mathrm{DR}}(z)$, and $\mathcal{P}_{g g}^{\mathrm{DR}}(z)$ in Eqs. $(38,39)$ are exactly the Altarelli-Parisi probabilities for the splitting processes $q \rightarrow q(z)+\phi(1-z), \quad q \rightarrow \phi(z)$ $+q(1-z)$, and $g \rightarrow \phi(z)+\phi(1-z)$, where $\phi$ is a scalar gluon.

Finally, we recall that setting $C_{F}=T_{R}=C_{A}$ in a tree-level QCD calculation leads to recovering the results in $N=1$ supersymmetric Yang-Mills theory [31]. The quark is replaced by the gluino $\widetilde{g}$ and the corresponding four-dimensional Altarelli-Parisi probabilities fulfill a well-known supersymmetric Ward identity, namely, $P_{g g}(z)+P_{g \widehat{g}}(z)=P_{\widetilde{g g}}(z)$ $+P_{\widetilde{g} \vec{g}}(z)$. As for the $d$-dimensional Altarelli-Parisi splitting functions in Eqs. (32)-(35), their version in conventional dimensional regularization violates a similar Ward identity. Using dimensional reduction, instead, we have

$\left\langle\hat{P}_{g g}^{\mathrm{DR}}(z ; \epsilon)\right\rangle+\left\langle\hat{P}_{g \widetilde{g}}^{\mathrm{DR}}(z ; \epsilon)\right\rangle=\left\langle\hat{P}_{\widetilde{g} g}^{\mathrm{DR}}(z ; \epsilon)\right\rangle+\left\langle\hat{P}_{\widetilde{g} \widetilde{g}}^{\mathrm{DR}}(z ; \epsilon)\right\rangle$,

thus leading to equal decay probabilities for the two supersymmetric partners in any number $d$ of space-time dimensions. This result is expected for a supersymmetric regularization.

We note that in the toy scheme, in which the number of quark states is analytically continued to be the same as the number of gluon states $h_{q}=h_{g}=2-2 \epsilon$, the same Ward identity is also recovered:

$\left\langle\hat{P}_{g g}^{\text {toy }}(z ; \epsilon)\right\rangle+\left\langle\hat{P}_{g \widetilde{g}}^{\text {toy }}(z ; \epsilon)\right\rangle=\left\langle\hat{P}_{\widetilde{g} g}^{\text {toy }}(z ; \epsilon)\right\rangle+\left\langle\hat{P}_{\widetilde{g} \widetilde{g}}^{\text {toy }}(z ; \epsilon)\right\rangle$.

\section{The master function $\mathcal{F}$ and $\mathrm{RS}$ independence of the cross sections}

The terms of order $\epsilon$, which arise in the splitting functions computed with different regularization prescriptions, combine with $1 / \epsilon$ poles coming from collinear singularities in the integrals of the dipole factors and thus provide the insertion operator $I^{\mathrm{RS}}$ in Eq. (15) with finite corrections.

More precisely, as discussed in the previous subsection, the RS dependence of the insertion operator $\mathbf{I}^{\mathrm{RS}}$ in dimensional regularization is entirely taken into account by the flavor functions $\mathcal{V}_{I}^{\mathrm{RS}}(\epsilon)$. The coefficients of the $\epsilon$ expansion of the functions $\mathcal{V}_{I}^{\mathrm{RS}}(\epsilon)$ are related to $d$-dimensional integrals of the splitting functions, as in Eq. (21). Different regularization prescriptions lead to contributions of the order of $\epsilon$ to the azimuthally averaged splitting functions $\left\langle\hat{P}_{a b}^{R S}(z ; \epsilon)\right\rangle$ and, in turn, to Eq. (21). These contributions produce a schemedependent shift in the constants $K_{a}$ on the right-hand side of Eq. (21) and, hence, nonsingular corrections in $\mathcal{V}_{I}^{\mathrm{RS}}(\epsilon)$. Pa- rametrizing these corrections as differences with respect to the flavor functions $\mathcal{V}_{I}(\epsilon)$ in conventional dimensional regularization, we have

$$
\mathcal{V}_{I}^{\mathrm{RS}}(\epsilon)=\mathcal{V}_{I}(\epsilon)-\widetilde{\gamma}_{I}^{\mathrm{RS}}+O(\epsilon),
$$

where the scheme-dependent coefficients $\widetilde{\gamma}_{I}^{\mathrm{RS}}$ are obtained by inserting Eq. (36) into Eq. (21),

$$
\widetilde{\gamma}_{a}^{\mathrm{RS}}=\sum_{b} \int_{0}^{1} d z \mathcal{P}_{a b}^{\mathrm{RS}}(z)
$$

Using Eq. (43) one can straightforwardly evaluate $\widetilde{\gamma}_{I}^{\mathrm{RS}}$ in different RS's. In particular, we have

$$
\widetilde{\gamma}_{I}^{\mathrm{HV}}=0,
$$

and, using Eq. (37) and Eqs. (38) and (39), we, respectively, find

$$
\begin{aligned}
& \widetilde{\gamma}_{q}^{\text {toy }}=\widetilde{\gamma}_{\bar{q}}^{\text {toy }}=0, \quad \widetilde{\gamma}_{g}^{\text {toy }}=-\frac{2}{3} T_{R} N_{f}, \\
& \widetilde{\gamma}_{q}^{\mathrm{DR}}=\widetilde{\gamma}_{\bar{q}}^{\mathrm{DR}}=\frac{1}{2} C_{F}, \quad \widetilde{\gamma}_{g}^{\mathrm{DR}}=\frac{1}{6} C_{A} .
\end{aligned}
$$

Equation (42) can be inserted in Eq. (31) to derive a simple expression for the RS dependence of the operator $\mathrm{I}^{\mathrm{RS}}$ in dimensional regularization. As a matter of fact, neglecting $O(\epsilon)$ corrections and using color-charge conservation $\sum_{J \neq I} \mathbf{T}_{J}=-\mathbf{T}_{I}$ (the insertion operator acts onto the vector $|1, \ldots, n\rangle_{\mathrm{RS}}$, which is a color-singlet state and, hence, fulfills the property $\Sigma_{J} \mathbf{T}_{J}|1, \ldots, n\rangle_{\mathrm{RS}}=0$ ), we obtain

$$
\mathbf{I}^{\mathrm{RS}}(\{p\}, \boldsymbol{\epsilon})=\mathbf{I}(\{p\}, \boldsymbol{\epsilon})-\frac{\alpha_{S}}{2 \pi} \sum_{I} \widetilde{\gamma}_{I}^{\mathrm{RS}}+O(\boldsymbol{\epsilon}) .
$$

In terms of the master function in Eq. (15), we can write

$$
\begin{aligned}
\mathcal{F}(\{p\})= & \left|\mathcal{M}^{\mathrm{RS}}(\{p\})\right|_{1 \text { loop }}^{2}-\frac{\alpha_{S}}{2 \pi}\left|\mathcal{M}^{\mathrm{RS}}(\{p\})\right|^{2} \sum_{I} \widetilde{\gamma}_{I}^{\mathrm{RS}} \\
& +\left|\mathcal{M}^{\mathrm{RS}}(\{p\})\right|^{2} \otimes \mathbf{I}(\{p\}, \boldsymbol{\epsilon}) .
\end{aligned}
$$

As usual, $\mathbf{I}(\{p\}, \epsilon)$ refers to conventional dimensional regularization and is given in Eq. (19).

Let us first comment on the result in Eq. (48) in the context of the toy scheme in which $h_{q}=2-2 \epsilon$. The renormalization of the ultraviolet divergences in the off-shell loop amplitudes is performed exactly as in conventional dimensional regularization. The only difference regards the definition of the running coupling. Using modified minimal subtraction, one introduces a renormalized coupling $\alpha_{S}^{\text {toy }}$ that is related to the customary $\overline{\mathrm{MS}}$ coupling by $\alpha_{S}^{\text {toy }}=\alpha_{S}[1$ $\left.-\alpha_{S} T_{R} N_{f} / 3 \pi+O\left(\alpha_{S}^{2}\right)\right]$. This is the harmless dependence on the ultraviolet regularization: the only physical consequence in an overall redefinition of the coupling constant. As soon as we consider the renormalized on-shell one-loop amplitude on the right-hand side of Eq. (48), we encounter soft and collinear divergences that are cancelled by the insertion operator $\mathbf{I}(\{p\}, \boldsymbol{\epsilon})$, computed in conventional dimensional regularization. However, according to our calculation, the remaining contribution has still to be corrected by the finite $\widetilde{\gamma}_{I}^{\text {toy }}$ terms in order to give a function $\mathcal{F}$ consistent with unitarity and, 
hence, RS-independent cross sections. This additional unitarity correction does depend on the process through the treelevel matrix element and its flavour topology $\left(\widetilde{\gamma}_{q}^{\text {toy }} \neq \widetilde{\gamma}_{g}^{\text {toy }}\right)$.

In the context of the 't Hooft-Veltman and dimensionalreduction prescriptions, the scheme dependence of the oneloop QCD amplitudes was studied in Ref. [16]. On the basis of the explicit evaluation of the one-loop corrections to all $2 \rightarrow 2$ QCD subprocesses, transition rules to relate these schemes to conventional dimensional regularization were derived (and argued to be universal). They were confirmed by the calculation in Ref. [10]. We can use these rules as a consistency check of our calculation and, in general, of our unitarization recipe. When inserted in Eq. (48), the coefficients $\widetilde{\gamma}_{I}^{\mathrm{DR}}$ in Eq. (46) provide the master function $\mathcal{F}$ with the contributions that are necessary to exactly cancel the RS dependence of the one-loop amplitudes $\left|\mathcal{M}^{\mathrm{RS}}(\{p\})\right|_{1 \text { loop }}^{2}$ as computed in Ref. [16].

We can turn this argument around. Writing Eq. (48) as

$$
\begin{aligned}
\left|\mathcal{M}^{\mathrm{RS}}(\{p\})\right|_{1 \text { loop }}^{2}= & -\left|\mathcal{M}^{\mathrm{RS}}(\{p\})\right|^{2} \otimes \mathbf{I}(\{p\}, \boldsymbol{\epsilon}) \\
& +\frac{\alpha_{S}}{2 \pi}\left|\mathcal{M}^{\mathrm{RS}}(\{p\})\right|^{2} \sum_{I} \widetilde{\gamma}_{I}^{\mathrm{RS}}+\mathcal{F}(\{p\}),
\end{aligned}
$$

and knowing that the function $\mathcal{F}$ is RS independent, we can compute the scheme dependence of the one-loop amplitudes $\left|\mathcal{M}^{\mathrm{RS}}(\{p\})\right|_{1 \text { loop }}^{2}$ from our calculation of the coefficients $\vec{\gamma}_{I}^{\mathrm{RS}}$. This is a way to rederive the transition rules found in Ref. [16]. Since the $\widetilde{\gamma}_{I}^{\mathrm{RS}}$, s are obtained from the integral of the dipole factors, this derivation is universal (the dipole factors do not depend on any specific QCD amplitude) and essentially involve a tree-level calculation (the emitted partons in the dipole are on shell) instead of a loop one.

Concluding this section on dimensional regularization, we should add a marginal comment. The RS dependence of the Altarelli-Parisi splitting functions may, in principle, also affect the factorization-scheme dependence of the NLO partonic cross sections if the collinear counterterm in Eq. (4) is not defined accordingly (for instance, if, independently of the regularization procedure, one uses the same minimally subtracted expression for $d \sigma_{C}$ ). In the notation of Eq. (7), this dependence is embodied in the contribution $\hat{\sigma}^{\mathrm{NLO}\{m\}}$ and, in particular, within the dipole algorithm, it is explicitly taken into account by the color-charge insertion operators $\mathbf{K}$ and $\mathbf{H}$ of Ref. [6]. If one is interested in changing factorization scheme, $\hat{\sigma}^{\mathrm{NLO}\{m\}}$ (e.g., the operators $\mathbf{K}$ and $\mathbf{H}$ ) and the parton distributions $f$ and $d$ in Eq. (1) have to be varied consistently. We think that pursuing the study of different $d$-dimensional factorization prescriptions has no particular relevance, in practice.

\section{CONCLUSION}

In this paper we have considered some unitarity issues related to the regularization of unphysical soft and collinear divergences in perturbative QCD computations. Our analysis has been performed in the framework of the dipole formalism. It allows one to work out a general discussion while providing an explicit implementation of unitarity constraints in the calculation of QCD cross sections at NLO.

In Sec. III we have shown that the regularization-scheme independence of physical observables is controlled by the master function

$$
\mathcal{F}(\{p\})=\left|\mathcal{M}^{\mathrm{RS}}(\{p\})\right|_{1 \text { loop }}^{2}+\left|\mathcal{M}^{\mathrm{RS}}(\{p\})\right|^{2} \otimes \mathbf{I}^{\mathrm{RS}}(\{p\}),
$$

which involves the one-loop and tree-level matrix elements and the integral $\mathbf{I}^{\mathrm{RS}}(\{p\})$ of the dipole factors. The latter are obtained by the factorization formula for soft and collinear emission. Using a unitary regularization scheme, infrared singularities as well as finite scheme-dependent remainders consistently cancel by combining the several contributions on the right-hand side of Eq. (50).

This result can also be used to relate one-loop amplitudes in different regularization schemes without explicitly carrying out any loop calculations. Turning Eq. (50) round,

$$
\left|\mathcal{M}^{\mathrm{RS}}(\{p\})\right|_{1 \text { loop }}^{2}=-\left|\mathcal{M}^{\mathrm{RS}}(\{p\})\right|^{2} \otimes \mathbf{I}^{\mathrm{RS}}(\{p\})+\mathcal{F}(\{p\}),
$$

and exploiting the scheme independence of $\mathcal{F}(\{p\})$, one can obtain $\left|\mathcal{M}^{\mathrm{RS}}(\{p\})\right|_{1 \text { loop }}^{2}$ in a different scheme by simply evaluating the difference of the corresponding dipole factors.

More generally, our explicit construction of Eq. (50) allows one to compute physical cross sections also using regularization prescriptions that are not manifestly unitary. As long as the regularization procedure is consistently defined at the level of one-loop amplitudes, we can give a recipe (see Sec. III) to calculate the dipole-factor contributions that are necessary to guarantee unitarity. From the regularized virtual corrections one can thus extract the essential physical information, that is, the regularization-scheme-independent function $\mathcal{F}(\{p\})$.

In order to make our general discussion more definite we have considered, in Sec. IV, the case of dimensionalregularization prescriptions. This analysis required the explicit calculation of the $d$-dimensional Altarelli-Parisi splitting functions in various regularization schemes. Our results for these functions differ from previously published ones in the 't Hooft-Veltman and dimensional reduction schemes $[2,16]$. These differences emerge in the $\epsilon$-dependent terms as can be seen by comparing our formulas (36)-(39) with the corresponding results in Refs. [2,16]. The differences come about because in these references the average over polarizations of the parent parton was taken, instead of the azimuthal average. These are not the same in the case of gluon splitting functions as discussed in Sec. IV C. However, this slight error has no practical consequence on the main results of Refs. $[2,16]$ because in those references the relationship between splitting functions and RS dependence was not exploited (in Ref. [16], the splitting functions were used to relate the definition of different factorization schemes). On the contrary, we make full use of this relationship, allowing us to derive the unitarity corrections needed to relate different dimensional-regularization schemes [see Eq. (49)], without having to make any loop calculations. Our results are in full agreement with those obtained in Ref. [16]. We are able to give a probabilistic interpretation [see Eq. (43)] of the coefficients $\tilde{\gamma}_{I}^{\mathrm{RS}}$. 
The method can also be applied to other regularization procedures, such as, for instance, massive regularization of the loop integrals. Many higher-order calculations in QED have been carried out using this regularization scheme. Dimensional regularization is certainly preferred in massless QCD and its computational advantages have also been exploited in recent QED achievements [32]. Our unitarization technique and the extension of the dipole formalism to massive partons [33] can be convenient in order to use known QED results in QCD applications and to combine them with new QED calculations in dimensional regularization.

Systematic QCD calculations at next-to-next-to-leading order (NNLO) for jet observables will become feasible only when efficient techniques for evaluating two-loop matrix elements will be set up. At that point the unitarity issue discussed in this paper will show up again. Our results may eventually be very useful to tackle this issue, provided the validity of the dipole formalism is extended to such a level of accuracy.

In concluding, we would like to point out another feature of Eq. (50). The operator $\mathbf{I}^{\mathrm{RS}}(\{p\})$ is obtained by integrating the dipole factors and, correspondingly, $\left|\mathcal{M}^{\mathrm{RS}}(\{p\})\right|_{1 \text { loop }}^{2}$ is the result of the integration over the loop momentum

$$
\begin{aligned}
\mathcal{F}(\{p\})= & \int_{\text {loop }} d\left|\mathcal{M}^{\mathrm{RS}}(\{p\})\right|_{1}^{2} \text { loop } \\
& +\sum_{\text {dipoles }} \int_{1}\left|\mathcal{M}^{\mathrm{RS}}(\{p\})\right|^{2} \otimes d V_{\text {dipole }}^{\mathrm{RS}}
\end{aligned}
$$

It is conceivable that one may find a way of combining the two integrands such that the dipole factors act as a local counterterm for the loop integral. Achieving this, one could avoid the introduction of any soft and collinear regularization and the ensuing unitarity problems. Most importantly, one would be able to carry out NLO calculations of physical cross sections by the sole use of numerical methods, without any analytical calculation of one-loop amplitudes.

\section{ACKNOWLEDGMENTS}

This research was supported in part by EEC Programme Human Capital and Mobility, Network Physics at High Energy Colliders, Contract Nos. CHRX-CT93-0357 (DG 12 COMA) and PECO ERBCIPDCT 94 0613, the Hungarian Science Foundation Grant No. OTKA T-016613, and the Research Group in Physics of the Hungarian Academy of Sciences, Debrecen.
[1] Z. Bern, L. Dixon, and D. A. Kosower, Annu. Rev. Nucl. Part. Sci. 46, 109 (1996), and references therein.

[2] W. T. Giele and E. W. N. Glover, Phys. Rev. D 46, 1980 (1992); W. T. Giele, E. W. N. Glover, and D. A. Kosower, Nucl. Phys. B403, 633 (1993).

[3] Z. Kunszt and D. E. Soper, Phys. Rev. D 46, 192 (1992); S. Frixione, M. L. Mangano, P. Nason, and G. Ridolfi, Nucl. Phys. B412, 225 (1994).

[4] S. Frixione, Z. Kunszt, and A. Signer, Nucl. Phys. B467, 399 (1996).

[5] S. Catani and M. H. Seymour, Phys. Lett. B 378, 287 (1996); in $Q C D$ and QED in Higher Orders, Proceedings of the Workshop, Rheinsberg Germany, 1996, edited by F. Blumlein et al. [Nucl. Phys. B (Proc. Suppl.) 51C, 233 (1996)], hep-ph/9607318.

[6] S. Catani and M. H. Seymour, Nucl. Phys. B485, 291 (1997).

[7] Z. Nagy and Z. Trócsányi, Nucl. Phys. B486, 189 (1997).

[8] Z. Bern, L. Dixon, and D. A. Kosower, Phys. Rev. Lett. 70, 2677 (1993).

[9] Z. Kunszt, A. Signer, and Z. Trócsányi, Phys. Lett. B 336, 529 (1994).

[10] Z. Bern, L. Dixon, and D. A. Kosower, Nucl. Phys. B437, 259 (1995).

[11] Z. Trócsányi, Phys. Rev. Lett. 77, 2182 (1996).

[12] W. B. Kilgore, Report No. Fermilab-Conf-96/315-T, hep-ph/9609367 (unpublished); W. B. Kilgore and W. T. Giele, this issue, Phys. Rev. D 55, 7182 (1997).

[13] A. Signer and L. Dixon, Phys. Rev. Lett. 78, 811 (1997).

[14] E. W. N. Glover and D. J. Miller, Durham Report No. DTP/ 96/06, hep-ph/9609474 (unpublished); Z. Bern, L. Dixon, D. A. Kosower, and S. Weinzierl, Nucl. Phys. B489, 3 (1997).

[15] W. Siegel, Phys. Lett. 84B, 193 (1979).
[16] Z. Kunszt, A. Signer, and Z. Trócsányi, Nucl. Phys. B411, 397 (1994).

[17] J. A. M. Vermaseren, K. J. F. Gaemers, and S. J. Oldham, Nucl. Phys. B187, 301 (1981).

[18] F. Bloch and A. Nordsieck, Phys. Rev. 52, 54 (1937); T. Kinoshita, J. Math. Phys. (N.Y.) 3, 650 (1962); T. D. Lee and M. Nauenberg, Phys. Rev. 133, B1549 (1964).

[19] See, for instance, A. Bassetto, M. Ciafaloni, and G. Marchesini, Phys. Rep. 100, 201 (1983); Yu. L. Dokshitzer, V. A. Khoze, A. H. Mueller, and S. I. Troyan, Basics of Perturbative QCD (Editions Frontières, Paris, 1991).

[20] R. K. Ellis, D. A. Ross, and A. E. Terrano, Nucl. Phys. B178, 421 (1981).

[21] R. P. Feynman, Acta Phys. Pol. 26, 697 (1963).

[22] G. 't Hooft and M. Veltman, Nucl. Phys. B44, 189 (1972).

[23] G. Bollini and J. J. Giambiagi, Nuovo Cimento B 12, 20 (1972); J. F. Ashmore, Lett. Nuovo Cimento 4, 289 (1972); G. M. Cicuta and E. Montaldi, ibid. 4, 329 (1972).

[24] R. Gastmans and R. Meuldermans, Nucl. Phys. B63, 277 (1973).

[25] W. Siegel, Phys. Lett. 94B, 37 (1980); L. V. Avdeev and A. A. Vladimirov, Nucl. Phys. B219, 262 (1983).

[26] D. M. Capper, D. R. T. Jones, and P. van Nieuwenhuizen, Nucl. Phys. B167, 479 (1980).

[27] I. Antoniadis and E. G. Floratos, Nucl. Phys. B191, 217 (1981).

[28] Z. Bern and D. A. Kosower, Nucl. Phys. B379, 451 (1992).

[29] G. Altarelli, R. K. Ellis, and G. Martinelli, Nucl. Phys. B157, 461 (1979).

[30] G. Altarelli and G. Parisi, Nucl. Phys. B126, 298 (1977).

[31] J. Wess and B. Zumino, Nucl. Phys. B70, 39 (1974).

[32] S. Laporta and E. Remiddi, Phys. Lett. B 379, 283 (1996).

[33] S. Catani, M. H. Seymour, and Z. Trócsányi (in preparation). 